\title{
LUSIN SETS AND WELL ORDERING THE CONTINUUM 1
}

\section{DAVID PINCUS AND KAREL PRIKRY}

ABSTRACT. A Lusin set is constructed in a model of set theory which lacks a well ordering of the continuum.

I. Introduction. A Lusin set is a subset $B \subset \mathbf{R}$ such that $|B|=2^{\boldsymbol{N}_{0}}$ and $|B \cap X| \leq \kappa_{0}^{2}$ for any $X$ of the first Baire category. A Lusin set can be proved to exist from the continuum hypothesis and the existence of a well ordering of the continuum. We shall show that neither of the above conditions is necessary for the existence of a Lusin set. More precisely:

Theorem. Let $M$ be a model of ZFC.3 Let $H$ be the Cohen-HalpernLévy model described in \$II. If a Lusin set exists in M then a Lusin set exists in $H$.

The theorem establishes the nonnecessity of the conditions since the continuum cannot be well ordered in $H$ and, by a suitable choice of $M$, the continuum hypothesis can be as false as one could wish (see Remarks III.1 and III.2). $\$$ II consists of a proof of the above theorem. $\$$ III is a discussion of the existence of other pathological linear sets in models of set theory lacking a well ordering of the continuum.

II. A Lusin set in $H$. Let $M$ be a fixed model of ZFC and let $A$ be a fixed Lusin set in M. The Cohen-Halpern-Lévy model $H(M)$ (or simply $H$ ) is constructed as follows.

Fix $\Phi \in M$ such that $\Phi$ is a 1-1 onto function from $\omega \times \omega$ to $\omega$. Let $a \in 2^{\omega}$ be Cohen-generic [9, p. 33] over M. In $M(a)$ define $a_{i} \in 2^{\omega}$ via $a_{i}(j)=$ $a(\Phi(i, j))$. Set $D=\left\{a_{i}: i \in \omega\right\}$ and $H=M(D \cup\{D\})$. Clearly $M \subset H \subset M(a)$.

Received by the editors August 8, 1973.

AMS (MOS) subject clas sifications (1970). Primary 02K05.

Key words and phrases. Lusin set, well ordering the continuum.

1 Research supported by NSF GP-44014.

$2 x_{\alpha}$ is the ath infinite well ordered cardinal. $2^{N_{0}}$ is the cardinal of the continuum, R. $|X|$ is the cardinal number of $X$.

$3 \mathrm{ZF}$ denotes the usual Zermelo-Fraenkel set theory. All models mentioned here are models of ZF. ZFC denotes Zermelo-Fraenkel set theory with choice. 
Alternative descriptions of $H$ can be found in [1, pp. 136-141] and [4]. From these sources:

II.1. $\mathbf{R}$ cannot be well ordered in $H$.

II.2. $\left(2^{\boldsymbol{N}_{0}}\right)^{M}=(\boldsymbol{x}(\mathbf{R}))^{H}$ and $\mathrm{Cf}^{M}(\alpha)=\mathrm{Cf}^{H}(\alpha) .4$

II.3. The axiom of choice for families of well orderable sets holds in H.

The Lusin set $B$ of $H$ is described quite simply in terms of $A$. Identify each $a_{i} \in D$ with the real number whose decimal expansion is $a_{i}$. If $G \in D^{*}$, the set of finite subsets of $D$, write

$$
{ }^{r} G=\left(a_{i_{1}}+a_{i_{2}}+\cdots+a_{i_{n}}\right) \bmod 1, \quad r_{\phi}=0,
$$

where the $a_{i_{j}}$ are the distinct members of $G$. Set:

$$
A_{G}=A+r_{G}=\left\{x+r_{G}: x \in A\right\}, \quad B=\bigcup_{G \in D^{*}} A_{G^{*}}
$$

It is clear that the definition of $B$ can be carried out in $H$. It remains to show that $B$ is a Lusin set in $H$. In preparation for this we develop below some more technical facts about generic reals and the Cohen-Halpern-Lévy model.

II.4. Every finite $G \subset D$ is a set of product generic Cohen reals [9, pp. 9-14]. $M\left(G_{2}\right)$.

II.5. If $G_{1}-G_{2} \neq \varnothing$ then any $x+r_{G_{1}}(x \in A)$ is Cohen-generic over

II. 5 follows from II. 4 together with the fact that ground model translates of a Cohen-generic real are Cohen-generic, and mod 1 sums of product generic Cohen reals are Cohen-generic.

II.6 (Immediate from II.5). If $G_{1} \neq G_{2}$ then $A_{G_{1}} \cap A_{G_{2}}=\varnothing$.

II.7 (From II.5 and the definition of Cohen-generic). For all closed nowhere dense $X \in \nabla G_{2}$ and for all $G_{1}$ such that $G_{1}-G_{2} \neq \varnothing, A_{G_{1}} \cap X=$ $\varnothing$.

In II. $\nabla G_{2}$ denotes the class of sets with codes in $M\left(G_{2}\right) \cdot \nabla G_{2}$ would be written $\bar{D}\left(G_{2}\right)$ in [1].

II.8. $|B|=2^{\boldsymbol{N}_{0}}$ in $H$.

$4 \boldsymbol{N}(X)$ denotes the least $\boldsymbol{N}_{\alpha}$ which is greater than or equal to the cardinal of every well ordered $Y \subset X$. Cf of the ordinal $a$ denotes the least ordinal cofinal with a. Superscripts denote relativization to the indicated classes. 
Proof. The exposition of $H$ in [1] yields that the equation $2^{\boldsymbol{X}_{0}}=$ $\left|D^{*} \times\left(2^{\boldsymbol{K}_{0}}\right)^{M}\right|$ holds in $H$. Let $f \in M$ be a $1-1$, onto mapping $\left(2^{\boldsymbol{N}_{0}}\right)^{M} \rightarrow A$. Define in $H g: D^{*} \times\left(2^{{ }^{*}}\right)^{M} \rightarrow B$ via $g(G, \alpha)=f(\alpha)+r_{G} \cdot g$ is $1-1$ and onto by II.6. This proves the lemma.

II.9. Lemma. " $|B \cap X| \leq \boldsymbol{\aleph}_{0}$ for first category $X "$ bolds in $H$.

Proof. Let $X$ be of the first category in $H$. We must show $|B \cap X| \leq$ $\boldsymbol{N}_{0}$ in $H$. By II.2 and II. 3 we may assume $X$ is closed and nowhere dense.

Fix $G_{0} \in D^{*}$ such that $X \in \nabla G_{0^{*}} . U=\mathbf{R}^{H}-X$ is also in $G_{0}$, hence so is $J=\left\{\langle r, s\rangle: r, s\right.$ rational and $\left.I_{r, s} \subset U\right\}$. Above $I_{r, s}$ denotes the open interval determined by $r$ and $s$.

$$
X \cap B=\bigcup_{G \in D^{*}}\left(A_{G} \cap X\right)=\bigcup_{G \subset G_{0}} A_{G} \cap X
$$

by II.7. Thus it suffices to show $\left|A_{G} \cap X\right| \leq \boldsymbol{\aleph}_{0}$ under the assumption $G \subset G_{0} \cdot\left|A_{G} \cap X\right| \leq \boldsymbol{\aleph}_{0}$ holds in $H$ iff $\left|A \cap X^{\prime}\right| \leq \boldsymbol{\kappa}_{0}$ holds in $H$ where $X^{\prime}=$ $\left\{x-r_{G}: x \in X\right\}$. Since $G \subset G_{0}, r_{G} \in \nabla G_{0}$ and $X^{\prime} \in \nabla G_{0^{\circ}} X^{\prime}$ is also closed and nowhere dense. Thus the problem for general $G \subset G_{0}$ is reduced to the problem for $G=\varnothing$.

For the closed, nowhere dense $X \in \nabla G_{0}$ set $X_{0}=X \cap M\left(G_{0}\right), U_{0}=$ $U \cap M\left(G_{0}\right)$. It is clear that $A \cap X=A \cap X_{0}$ and, by standard facts on coding [9], $X_{0}$ is closed and nowhere dense in $M\left(G_{0}\right)$. It therefore suffices to show that " $\left|A \cap X_{0}\right| \leq \boldsymbol{\aleph}_{0}$ " holds in $M\left(G_{0}\right)$.

This final step uses an argument of Silver which is exposited, for example, in [7]. We note first that $M\left(G_{0}\right)$ is a generic extension of $M$ with respect to a countable partially ordered set $(P, \leq)$. Arguing by contradiction assume " $\left|A \cap X_{0}\right| \geq \boldsymbol{\kappa}_{1}$ " holds in $M\left(G_{0}\right)$. There must be some "true" $p \in P$ which forces $\dot{x} \in A \cap \dot{X}$ ( $\dot{y}$ is a term representing $y$ ) for uncountably many $x \in A$. Below $\mathbb{f}$ denotes "forces".

$$
Y=\left\{x: p \Vdash \dot{x} \in A \cap \dot{X}_{0}\right\} \in M
$$

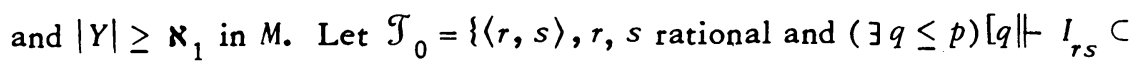
$\left.\left.U_{0}\right]\right\} . T=\bigcup_{\langle r, s\rangle \in \mathcal{T}_{0}}{ }{ }_{r s}$ is a dense open set in $M$ since $U_{0}$ is dense-open in $M\left(G_{0}\right) . T \cap Y=\varnothing$ since $U_{0} \cap Y=\varnothing$. Thus $Y \subset A \cap\left(\mathbf{R}^{M}-T\right)$ contradicting the fact that $A$ is Lusin in M. This completes the proof of the lemma and the theorem. 
III. Remarks. III.1. The following is shown in [10].

Theorem. Let $N$ be a model of $Z F C+2^{\boldsymbol{N}_{0}}=\boldsymbol{X}_{1}$. Let $\lambda \in N$ be a cardinal not cofinal with $\omega$ in $N$. There is a Cohen extension $M$ of $N$ preserving cofinalities, having a Lusin set, and in which $2^{\boldsymbol{N}_{0}}=\lambda$.

This, together with our Theorem and II.2, gives

Corollary. Let $N$ be a model of $Z F C+2^{\boldsymbol{N}_{0}}=\boldsymbol{\kappa}_{1}$. Let $\lambda \in N$ be a cardinal not cofinal with $\omega$ in $N$. There is an extension $H$ of $N$ preserving cofinalities, having a Lusin set, satisfying $\boldsymbol{\kappa}(\mathbf{R})=\lambda$, and having no well ordering of $\mathbf{R}$.

III.2. It is remarked in [2] that for $N$ a model of $\mathrm{ZFC}+\mathrm{GCH}$ and any $N$-cardinal $\lambda>\omega$ one can find an extension $H$ of $N$ preserving cofinalities and satisfying $\boldsymbol{\kappa}(\mathbf{R})=\lambda$. One might thus hope to drop the hypothesis that $\lambda$ is cofinal with $\omega$ in the corollary of III.1. This is possible as we outline below for the case $\lambda=\boldsymbol{N}_{\omega}$.

For each $n \in \omega$ let $D_{n}$ be an independent set of $\boldsymbol{x}_{n}$-sequences of independent Cohen reals (see [1, pp. 129-134]). Set $H^{*}=N\left[\left\{D_{n}: n \in \omega\right\}\right]$. For a finite $G \subset D_{0} \cup \ldots \cup D_{n}(n$ least $)$ and $\alpha<\boldsymbol{x}_{n}$ define

$$
r_{G}^{\alpha}=a_{i_{1}}^{0}+\cdots+a_{i_{k}}^{0}+a_{j_{1}}^{\alpha}+\cdots+a_{j_{l}}^{\alpha}
$$

where $i_{1}, \ldots, i_{k}$ are indices of sequences in $D_{0} \cup \ldots \cup D_{n-1}$ and $j_{1}, \ldots$, $j_{l}$ are indices of sequences in $D_{n}$. Set

$$
B=\bigcup_{G} \bigcup_{a<\boldsymbol{N}_{n}} A+r_{G}^{a}
$$

where $A$ is a Lusin set in $L$. The arguments of $\oint I I$ prove that $B$ is a Lusin set in $H^{*}$. Lemma II.9 works because every closed nowhere dense $X \in H^{*}$ is coded in some $L(b), b \in H^{*}, b$ Cohen generic, by a countable chain condition argument.

Remarks III. 1 and III. 2 form the basis for the assertion that our theorem permits the continuum hypothesis to be "as false as one could wish." It is also possible (as in the model of [9]) to have a non-well orderable continuum together with the continuum hypothesis in the form $\sim \exists_{M}\left[\boldsymbol{x}_{0}<M<2^{\boldsymbol{x}_{0}}\right]$. We do not know whether a Lusin set can exist under such conditions. Remark III.3(a) below implies that no Lusin set exists in the model of [9].

III.3. We list some other sets which exist in $H$. 
(a) Nonperfect set. This is a $B \subset \mathbf{R}$ such that $|B|=2^{\boldsymbol{N}_{0}}$ and any closed $E \subset B$ satisfies $|E| \leq \boldsymbol{\aleph}_{0}$. A Lusin set is nonperfect ([5] gives a proof of this without resort to the axiom of choice). The existence of a nonperfect set can be established by reworking $\$ I I$ even if a Lusin set does not exist in $M$.

(b) Nonprincipal ultrafilter on $\mathcal{P}(\omega)$. This exists by the prime ideal theorem for Boolean algebras, which holds in $H$ by [4]. Its image under the "binary decimal" map to the unit interval is a set which is nonmeasurable and without the Baire property (see the introduction to [6]).

(c) Vitali set. This is a choice function on the set of equivalence classes of reals under the relation " $x \sim y \leftrightarrow x-y$ is rational".

A Vitali set is well known to be nonmeasurable and without the Baire property. Its existence is clear from II. 3 and was first remarked by Feferman.

We would be interested in knowing whether a Hamel basis for $\mathbf{R}$ over $Q$ (the rationals) exists in $H$ or in any other model in which $\mathbf{R}$ cannot be well ordered。

III.4. The Feferman model, $F$, is the subclass of $H$ consisting of sets hereditarily definable in $H$ using parameters in $\mathbf{R} \cup M .5$ None of the sets discussed in III. 3 exists in $F$.

That no nonperfect set exists in $F$ was recently communicated to us by John Truss. Feferman [3] proved that no nonprincipal ultrafilter on $\mathscr{P}(\omega)$ exists in $F$ and stated that Scott had shown the nonexistence of a Vitali set in $F$. The nonexistence of a Hamel basis is established similarly. The existence of a Hamel basis implies the existence of an additive function $f: \mathbf{R} \rightarrow Q$ such that $f$ is the identity on $Q$. Symmetry considerations show, however, that if $x$ is generic over the definition of $f$ and $q \in Q$ is such that $0<q$ and $q$ is sufficiently small then $f(x+q)=f(x)$.

On the other hand none of the three main results in the model of [9] hold for $F . \boldsymbol{K}_{1}<2^{\boldsymbol{N}_{0}}$ in $F$ so there is an uncountable set with no perfect subset. Lemma II. 9 is used in [6] to remark that a set without the Baire property exists in $F$. It will be remarked in III. 6 that a nonmeasurable set exists in $F$.

III.5. A heuristic duality theory exists connecting notions having to do

5 This is a slight modification of the model studied in [3, p. 343]. It is dual to the model of Solovay studied in [8, \$4]. Consistencies established in $F$ do not depend on an inaccessible cardinal, unlike those of [9]. 
with Baire category and notions having to do with Lebesgue measure. Dual, in this sense, to a Lusin set is a co-Lusin set, a subset $B \subset \mathbf{R}$ such that $|B|=2^{\boldsymbol{N}_{0}}$ and $|B \cap X| \leq \boldsymbol{\kappa}_{0}$ for any $X$ of measure 0 . It is natural to ask whether a co-Lusin set exists in $H$ (when, let us say, a co-Lusin set exists in $M$ ). The answer is no. The key fact here, due to Solovay, was stated by Silver in unpublished lectures at the 1967 UCLA summer institute.

Fact. If $a$ is Cohen-generic there is no Solovay-generic (random see $[9$, p. 33]) real in $L(a)$.

Corollary. $\mathbf{R}^{H}$ is the union of null sets coded in $M$.

The corollary, together with II.1., II.2, and II.3 immediately implies the nonexistence of a co-Lusin set in $H$.

III.6. The following theorem was recently communicated to us by John Truss. DC denotes the axiom of dependent choice (see [9]).

Theorem $(\mathrm{ZF}+\mathrm{DC})$. If every set is Lebesgue measurable then Lebesgue measure is well ordered additive (i.e. $\boldsymbol{\kappa}_{a}$ additive for every ordinal a).

We deduce

Corollary $(\mathrm{ZF}+\mathrm{DC})$. If every set is Lebesgue measurable and $M$ is any transitive class modeling ZFC then all but a null set of reals are Solovay-generic over $M$.

DC holds in $F$ (dualizing the argument of [8, p. 407]). Thus by the corollary and the fact of III. 5 there is a nonmeasurable set in $F$.

We remark that the theorem and its corollary dualize according to the correspondence

Lebesgue measurable

Well ordered additive measure

Solovay-generic

Null set
Has Baire property

$\sim$ Well ordered Baire category the orem

$\sim$ Cohen-generic

$\sim$ First category set

III.7. A natural attempt to dualize the model $H$ is to form the model $H^{+}$ from $M(s), s$ Solovay-generic, in the same way as $H$ is formed from $M(a), a$ Cohen-generic. We found it easier to work with a model $\mathrm{H}^{++}$formed from $M(t), t$ a Solovay-generic subset of $\left|2^{K_{0}}\right|^{M}$, using a $\Phi$ pairing $\left|2^{N_{0}} \times 2^{N_{0}}\right|^{M}$ with $\left|2^{N_{0}}\right|^{M}$. 
An argument of Solovay shows that the support theory (see [1, p. 140]) works in $H^{+}$and $H^{++}$. Also, since III.5 dualizes, one has no Lusin set in $H^{+}$or $H^{++}$. We can obtain a co-Lusin set in $H^{++}$but we do not know about $H^{+}$. The prime ideal theorem, or even a nonprincipal ideal in $\mathcal{P}(\omega)$, is open in both $\mathrm{H}^{+}$and $\mathrm{H}^{++}$.

The reader is referred to $[8, \$ 4]$ for a discussion of the dual of $F$.

\section{BIBLIOGRAPHY}

1. P. J. Cohen, Set theory and the continuum hypothesis, Benjamin, New York, 1966. MR 38 \#999.

2. J. Derrick and F. Drake, A model of ZF set theory with $\boldsymbol{N}\left(2^{\omega}\right)=\boldsymbol{\aleph}_{\omega}, \mathrm{J}$. Symbolic Logic 32 (1967), 559. (abstract)

3. S. Feferman, Some applications of the notions of forcing and generic sets, Fund. Math. 56 (1964/65), 325-345. MR 31 \#1193.

4. J. D. Halpern and A. Lévy, The Boolean prime ideal theorem does not imply the axiom of choice, Proc. Sympos. Pure Math., vol. 13, part I, Amer. Math. Soc., Providence, R. I., 1971, pp. 83-134. MR 44 \#1557.

5. C. Kuratowski, Topologie. Vol. I, 2nd ed., Monografie Mat., Tom 20, PWN, Warsaw, 1948; English transl., new ed., Academic Press, New York; PWN, Warsaw, 1966. MR 10, 389; 36 \#40.

6. D. Pincus, The strength of the Hahn-Banach theorem, Proc. 1972 Victoria Sympos. on Nonstandard Analysis, Springer-Verlag, New York, 1974, pp. 203-248.

7. K. Prikry, Changing measurable into accessible cardinals, Dissertationes Math. Rozprawy Mat. 68 (1970). MR 41 \#6685.

8. G. E. Sacks, Measure-theoretic uniformity in recursion theory and set theory, Trans. Amer. Math. Soc. 142 (1969), 381-420. MR 40 \#7.108.

9. R. M. Solovay, A model of set-theory in which every set of reals is Lebesgue measurable, Ann. of Math. (2) 92 (1970), 1-56. MR 42 \#64.

10. P. Vopěnka and $\mathrm{K}$. Hrbáček, The consistency of some theorems on real numbers, Bull. Acad. Polon. Sci. Sér. Sci. Math. Astronom. Phys. 15 (1967), $107-$ 111. MR 37 \#1255.

DEPARTMENT OF MATHEMATICS, UNIVERSITY OF WASHINGTON, SEATTLE, WASHINGTON 98195

DEPARTMENT OF MATHEMATICS, UNIVERSITY OF MINNESOTA, MINNEAPOLIS, MINNESOTA 55455 